\title{
Técnicas de otimização em Aceleradores Vetoriais NEC SX-Aurora*
}

\author{
Félix D. P. Michels ${ }^{1}$, Matheus S. Serpa ${ }^{1}$, Danilo Carastan-Santos ${ }^{1}$ \\ Lucas M. Schnorr ${ }^{1}$, Philippe O. A. Navaux ${ }^{1}$ \\ ${ }^{1}$ Instituto de Informática - Universidade Federal do Rio Grande do Sul (UFRGS) \\ Caixa Postal 15.064 - 91.501-970, Porto Alegre - RS - Brasil \\ Programa de Pós-Graduação em Computação \\ \{felix.junior, msserpa, danilo.csantos, schnorr, navaux\}einf.ufrgs.br
}

\begin{abstract}
Resumo. Avalia-se nesse trabalho a utilização de técnicas de otimização clássicas na nova arquitetura NEC SX-Aurora. Utilizou-se como estudo de caso o benchmark NAS e uma aplicação real de migração sísmica, utilizada pela indústria de petróleo e gás. Os resultados experimentais finais mostram a melhora no desempenho, em FLOPS, utilizando as técnicas de otimização loop unrolling e inlining, no benchmark NAS em até 7,8× e na aplicação real de migração sísmica em até $1,9 \times$, em comparação com o desempenho das versões originais.
\end{abstract}

\section{Introdução}

A computação de alto desempenho provê diversos benefícios para as mais variadas áreas do conhecimento, como por exemplo, a análise de dados criando novas possibilidade com novas modelagens e simulações [Ezell and Atkinson 2016]. Diferentes tipos de arquiteturas são utilizados atualmente, tais como aceleradores vetoriais. Para aprimorar o desempenho, técnicas de otimização são empregadas. Técnicas para melhorar a vetorização e aumentar a taxa de acerto da memória cache são utilizadas regularmente.

Semelhante a este trabalho a publicação de Komatsu et al. mostra o potencial da arquitetura SX-Aurora TSUBASA. Uma comparação com outras arquiteturas, NVIDIA Tesla V100 e SX-Ace, entre outras, mostra resultados em que a SX-Aurora tem capacidade de executar eficientemente, com até 3,5× de desempenho, além de obter um speedup maior de até $2,8 \times$ [Komatsu et al. 2018].

Neste trabalho, otimiza-se o desempenho de uma aplicação real de Reverse Time Migration (RTM), utilizada na indústria de petróleo e acústica, e o conjunto de benchmarks NAS utilizando técnicas de loop unrolling e inlining na nova arquitetura da NEC, a SX-Aurora TSUBASA, procurando melhorar a vetorização automática desempenhada pelo compilador.

\section{Metodologia e Experimentos}

Para a avaliação da SX-Aurora, utilizou-se o benchmark NAS 3.4 e uma aplicação real utilizada pela indústria de petróleo para migração sísmica, denominada RTM. Utilizando

\footnotetext{
*Este trabalho foi parcialmente financiado com apoio da Coordenação de Aperfeiçoamento de Pessoal de Nível Superior - Brasil (CAPES) - Código de Financiamento 001, pelo projeto Petrobras (2016/00133-9, 2018/00263-5) e pelo projeto "GREEN-CLOUD: Computação em Cloud com Computação Sustentável” (\#16/2551-0000 488-9), da FAPERGS e do CNPq, programa PRONEX 12/2014.
} 
os contadores de hardware da máquina vetorial SX-Aurora, pode-se constatar uma baixa taxa de vetorização e uma alta taxa de erro da cache. Portanto, aplicou-se duas técnicas de otimização: inlining que substitui uma chamada de função pela própria função; loop unrolling que consiste em desenrolar laços. Utilizou-se o sistema operacional CentOS 7.9 e os compiladores exclusivos da NEC.

A execução dos experimentos utilizou os recursos da infraestrutura PCAD, http://gppd-hpc.inf.ufrgs.br, no INF/UFRGS, principalmente o ambiente SX-Aurora TSUBASA. O acelerador vetorial possui um processador de 8 cores a $1408 \mathrm{MHz}$, com memória global de $48 \mathrm{~GB}$ a $900 \mathrm{MHz}$, três níveis de cache, sendo a cache L1 e L2 para cada core e cache L3, uma unidade de processamento vetorial e uma unidade de processamento escalar [Komatsu et al. 2018]. Todos os experimentos foram executados 10 vezes, demonstrando os valores médios dessas execuções, utilizando uma distribuição de erro padrão.

\section{Resultados das otimizações e Conclusão}

A seguir são apresentados os resultados dos experimentos. Inicialmente, são expostos os resultados dos experimentos sendo a principal métrica avaliada a de operaçoes de ponto flutuante por segundo (FLOPS). Obtiveram-se os dados através de contadores de hardware do acelarador SX-Aurora, e para visualização dos dados uma rotina em $\mathrm{R}$ foi desenvolvida. Finalmente, os resultados obtidos são expostos, comparando o otimizado e original.

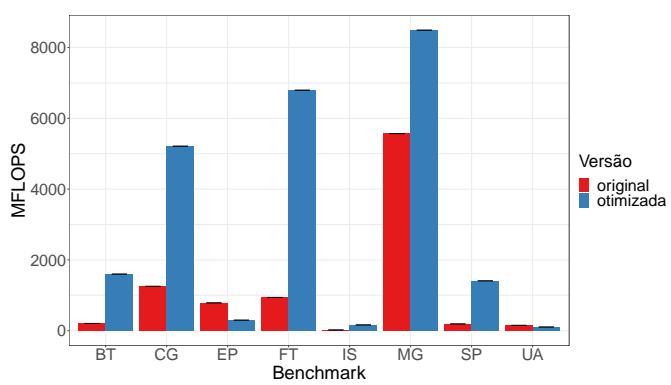

(a) NAS

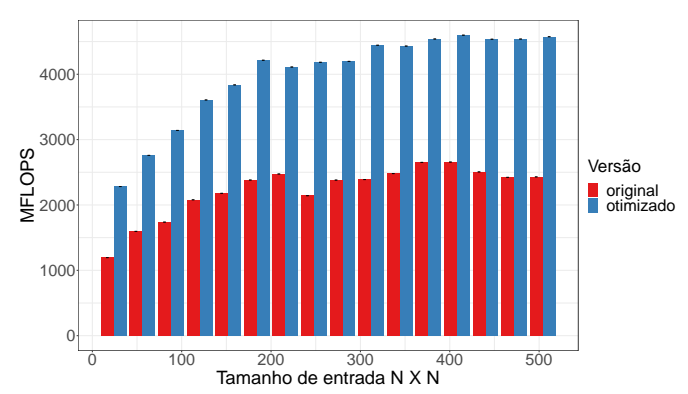

(b) RTM

Figura 1. MFLOPS X Tamanho da entrada

Percebe-se na Figura 1 que as técnicas influenciaram em um aumento em FLOPS considerável, chegando até $7,8 \times$ maior para a aplicação otimizada do benchmark NAS. Comparando o aumento entre a versão original e a otimizada, temos uma melhora máxima de, respectivamente, 204,42 MFLOPS e 1599,18 MFLOPS em referência ao benchmark BT. Para o RTM o aumento máximo foi de aproximadamente 1, $9 \times$, de 2429,83 MFLOPS para 4574,75 MFLOPS referindo-se ao tamanho de entrada de $504 \times 504$.

\section{Referências}

Ezell, S. J. and Atkinson, R. D. (2016). The vital importance of high-performance computing to us competitiveness. Information Technology and Innovation Foundation, April.

Komatsu, K., Momose, S., Isobe, Y., Watanabe, O., Musa, A., Yokokawa, M., Aoyama, T., Sato, M., and Kobayashi, H. (2018). Performance evaluation of a vector supercomputer sx-aurora tsubasa. In SC18: International Conference for High Performance Computing, Networking, Storage and Analysis, pages 685-696. IEEE. 